\title{
39th Meeting of the Pediatric Section of the German Society of Trauma Surgeons (DGU)
}

\author{
June 11-12, 2021 \\ Tübingen, Germany
}

(C) The Author(s) 2021

\author{
Scientific chair \\ PD Dr. med Justus Lieber \\ Children's University Hospital \\ Department of Pediatric Surgery \& Pediatric Urology \\ Tübingen \\ Dr. med. Hans-Joachim Kirschner \\ Children's University Hospital \\ Department of Pediatric Surgery \& Pediatric Urology \\ Tübingen
}

\section{PD Dr. med. Ralf Kraus}

Klinikum Bad Hersfeld

Clinic for Trauma Surgery \& Orthopedics

Bad Hersfeld

\footnotetext{
Sponsorship: This abstract publication was sponsored by the German Society of Trauma

Surgeons (DGU)
}

Review: The abstracts were selected and reviewed by Dr. med. Justus Lieber,

Dr. Hans-Joachim Kirschner \& Dr. med. Ralf Kraus. 
„To mend with a bend ": An easy way to fix distal radial shaft/forearm fractures

\section{Krohn}

Department of Pediatric Surgery, Klinikum Schwabing, Munich, Germany

Introduction: Distal radial shaft/forearm fractures are a challenge for osteosynthesis, because simple intramedullary nailing often results in a significant radial displacement of the distal radial fragment and $\mathrm{K}$-wires sometimes cannot be placed into the proximal fragment because the distal fragment is too long. However osteosynthesis with a plate or an external fixation device seems to be a procedure too invasive for such a „simple“ fracture. Therefore, the author developed an osteosynthesis with a double-bended intramedullary nail which achieves a minimal invasive osteosynthesis with anatomically correct reduction of the fracture.

Methods: An intramedullary nail (ESIN/TEN) is used and is bent about $45^{\circ}$ approximately $1 \mathrm{~cm}$ proximal to the fracture (before inserting it into the radius-radiologically controlled). About 2 or $3 \mathrm{~cm}$ further distally (depending on the size of the patient), it is bent again $45^{\circ}$ in the other way, so that a z-shaped intramedullary nail results. When placed into the radius, the nail is rotated in a way, that the first bend is placed on the proximal end of the fracture, radially and the second on the distal end of the fracture on the ulnar side. This leads to an anatomically correct reduction. If the ulna is fractured as well, it is stabilized with a normal intramedullary nail. However: This is not a classic intramedullary osteosynthesis, because now there are two hypomochlions which could lead to a flip of the nail. For that reason, we do recommend an immobilization in a plaster for 2 weeks after osteosynthesis.

Results: In all patients where we used this minimal invasive method, we could achieve an almost anatomically correct reduction of the fracture. A plate or an external fixation device was therefore never necessary.

Conclusion: „To mend with a bend" (,der Trick mit dem Knick“) is a minimal invasive and fast technique, that provides a very useful approach to the fracture of the distal radial shaft / forearm, and if this technique is performed the right way, this is not a problematic fracture anymore.

\section{Treatment of pediatric diametaphyseal forearm fractures: conclusions from 92 cases}

\section{Dietzel, S. Scherer, M. Schunn, H. J. Kirschner, J. Fuchs, J. Lieber}

Department of Pediatric Surgery and Pediatric Urology, Children's University Hospital, Tübingen, Germany

Question: Elastic stable intramedullary nailing is considered the standard treatment for displaced or unstable diaphyseal forearm fractures in children as is classic $\mathrm{K}$-wire for pediatric distal metaphyseal fractures. Until today there is only few data regarding diametaphyseal forearm fractures and no evidence-based treatment recommendations. Our objective was to collect data from pediatric forearm fractures during a 5-year period, focusing on diametaphyseal fractures. Situated between diaphyseal and distal fractures, they have shared features but also specific characteristics.

Methods: Retrospective data analysis of all pediatric forearm fractures at our center between January, 2015 and December, 2019, respectively. Breakdown to proximal, diaphyseal, diametaphyseal and distal lesions. Detailed review of all cases with diametaphyseal fractures. Exclusion of cases with primary operative treatment at other hospitals and of patients without follow-up.
Results: 1290 pediatric forearm fractures were treated at our center between 2015 and 2019 of which 92 were diametaphyseal, equaling $7 \%$ of all forearm fractures. Mean age at diagnosis was 7.6 years (11 months-14 years, median 7 years). Gender ratio was boys-girls 2:1. Ordinary falls were the most common accident cause followed by bicycle spills and sporting accidents. 58 diametaphyseal forearm fractures were treated conservatively, of which 23 underwent closed reduction and retention, 34 were immobilized only. In one case, Secondary displacement occurred 10 days after closed reduction and was then stabilized with ESIN. In the same patient, ESIN had to be removed followed by plate osteosynthesis due to inadequate bone position. 34 diametaphyseal fractures underwent direct operative therapy. Transepiphyseal intramedullary K-wire fixation was used in 22 cases, of which 14 received additional ESIN of the ulna. 7 fractures were primarily treated with ESIN and 4 cases with classic bicortical K-wire fixation (of which 2 receiving additional stabilization of the ulna). 1 case was treated with a plate. In 1 patient, elastic nails were removed and changed to plate fixation on short notice because of insufficient alignment. Complications and problems after surgery were re-fracture 1 month after implant removal $(n=1)$, pin site infection $(\mathrm{n}=1)$ and lesion of the skin covering the buried wire at the wrist $(n=1) .6$ cases had an event-free course with implant removal performed elsewhere or not carried out at all.

Conclusion: Diametaphyseal forearm fractures in children should be regarded as stand-alone injury between fractures of the shaft and the metaphysis. Therefore, it needs special consideration in conservative as well as operative therapy. Complication rates are low for conservatively treated fractures if stability is closely monitored using X-ray controls. For operative treatment of diametaphyseal forearm fractures in children, transepiphyseal intramedullary K-wire fixation is a safe option with few complications.

\section{Comparison of antegrade and retrograde flexible intramedullary nailing of forearm fractures at the distal metadiaphyseal junction in children}

S. Rohleder, O. Müller, M. Schwind, O. Muensterer

Department of Pediatric Surgery, University Medical Center, Mainz, Deutschland

Question: Treatment of unstable forearm fractures at the distal radius metadiaphyseal junction (DRMJ) in children presents a particular challenge. The ideal treatment of this fracture type has not yet been established. We investigated the use of the antegrade vs. the retrograde elastic stable intramedullary nailing (ESIN) technique for this fractures type.

Methods: From 2017-2019 all children until the age of 14 years with forearm fractures who were treated with flexible intramedullary nailing were included retrospectively. Postoperative fracture alignment and technical related complications for the retrograde, posteromedial and antegrade (Thompson) approach were analyzed.

Results: During observation period, a total of 133 radius fractures (median age 7.8 years) were treaded using the ESIN technique. Fractures were located at the DRMJ in $30.1 \%(n=40)$. A distal posteromedial entry at the Tuberculum listerii (group 1) was performed in $30 \%$ ( $\mathrm{n}=12$ ), an antegrade technique using the Thompson approach (group 2) in $15 \%(\mathrm{n}=6)$, and a standard lateral approach (group 3) was used in 55\% $(\mathrm{n}=22)$ cases. Persistent postoperative fracture angulation between $5^{\circ}$ and $10^{\circ}$ was noted in 3 cases in Group 1, 2 cases Group 2 and 9 cases Group 3. Extensor Pollicis Longus (EPL) tendon lesions were significantly associated with the posteromedial approach in Group $1(\mathrm{n}=3,25 \%, \mathrm{p}>0.01)$. 
Conclusion: Antegrade elastic stable intramedullary nail fixation for pediatric distal radius diaphyseal metaphyseal junction fractures is easily and securely achievable, without risking EPL tendon lesions.

\section{Nonunions after forearm fractures in children-a rare complication after fracture treatment}

\section{O. Loose, F. Fernandez}

Olgahospital, Stuttgart, Germany

Question: Diaphyseal forearm fractures occur frequently in children. The standard of care for displaced fractures is flexible intramedullary nailing. Non-union is a rare complication after surgical treatment, but can occur in both the radius or ulna. The aim of this study is to evaluate predisposing factors for non-union in these fractures.

Methods: We conducted a retrospective review of all patients who were treated primarily or secondary at our institution for non-union after flexible intramedullary nailing in the period from 1990 to 2018 . Non-union was defined as the lack of bony consolidation 6 months after fracture (8). In addition to epidemiological data, the cases were examined primarily with regard to the type of fracture, the geometry of the fracture, the localisation of the fracture, the associated soft tissue damage (open or closed) and possible postoperative complications or other associated factors.

Results: 13 cases were identified. Average age was 12 years (9-16 years). Ulna was affected in 11 cases and the radius in two. The most common location for ulna non-union was the middle third of the shaft. There were 11 closed and two open fractures. In ten cases an open reduction was required. In two cases technical problems were identified. In most cases (11/13) a hypertrophic non-union was identified. Indications for repeat surgery were increasing deformity, pain and limitation of movement.

Conclusion: Non-unions are a rare condition after intramedullary stabilisation of forearm fractures in children. The most affected region is the mid-shaft. Open reduction seems to be an influencing factor. Resection of non-union with cancellous bone plastic and plate osteosynthesis leads to healing.

\section{Avulsion injuries of the palmar portion of the proximal interphalangeal (PIP) joint-epidemiology, diagnosis and outcome}

\section{S. Deeg, L. M. Wessel, D. Svoboda}

Department of Pediatric Surgery, University Medical Center Mannheim, Mannheim, Germany

Question: Which trauma mechanisms lead to avulsion injuries of the palmar proximal interphalangeal (PIP) joint, what is the epidemiology of this injury in children and how should we treat it? What is the outcome after these injuries?

Methods: We retrospectively analyzed children with palmar fibrocartilage injuries of the proximal interphalangeal (PIP) joint treated at our institution in 2018. These patients came to our pediatric surgery department primarily as emergencies. If an injury to the palmar portion of the proximal interphalangeal joint (PIP joint) was suspected, then a conventional radiograph was performed. In the case of an avulsion fracture, we splinted for 1 week, after which the affected finger was solidarized with its neighbor using buddy tapes for approximately 2-3 weeks. In case of isolated ligamentous lesions in adolescence, these patients also received splinting for 5-10 days, followed by buddy tapes for 3 weeks. The final follow-up was after removal of the buddy tapes.

Results: In 2018, we treated 19 children with palmar fibrocartilage injuries of the proximal interphalangeal (PIP) joint, 9 boys and 10 girls. We had 12 avulsion fractures and 7 ligamentous lesions of the palmar fibrocartilage with an average age of 11 years. We also treated 3 patients with avulsion fractures of the phalangoglenoid ligament. Trauma mechanism was predominantly hyperextension during ball sports. All patients were fully convalescent. In one case of palmar fibrocartilage injury treated in another institution with consecutive unstable PIP joint, surgical revision was required in our pediatric hand surgery unit. Intraoperatively, the palmar fibrocartilage was mobilized and fixed using a transosseous suture.

Conclusion: If anamnesis and typical clinical features are known, injuries of the palmar fibrocartilage of the proximal interphalangeal (PIP) joint can be treated easily. The correct treatment leads to total recovery in all patients.

Conclusion: Avulsion injuries of the palmar aspect of the proximal interphalangeal (PIP) joint in children can be easily treated with splinting for 1 week followed by buddy taping for 3 weeks if the history and typical clinical features are known. Proper treatment leads to complete recovery in all patients.

\section{Long-term results after treatment of pediatric medial humeral epicondyle fractures with and without associated elbow dislocation}

\section{S. Scherer, M. Dietzel, N. Jordan, I. Tsiflikas*, H.-J. Kirschner, J. Fuchs, J. Lieber}

Department of Pediatric Surgery and Pediatric Urology, *Department of Diagnostic Radiology, Children's University Hospital, Tübingen, Germany

Question: Fractures of the medial humeral epicondyle are either isolated or associated with elbow dislocations. Treatment concepts are still a matter of debate. This single center study analyzes the own approach, shows changes of treatment over an observation period of 16 years, and reports long-term results.

Methods: Retrospective analysis of all patients treated with a medial humeral epicondyle fracture between 2005 and 2020 at our institution. Results: Ninety-six patients $(\varnothing 9.3$ years, range $1-15)$ were included in the study. In 25 cases (26\%), the fracture was associated with an elbow dislocation. The majority of patients received surgical treatment $(83.3 \%)$, whereas $17.7 \%$ were treated conservatively. Conservative treatment was performed using an above the elbow cast for a mean of 27.9 days (range 20-40). Surgical treatment consisted of open reduction and fixation with compression screw ( $n=44$ steel, $\mathrm{n}=2$ absorbable), K-wire $(\mathrm{n}=30)$, a combination of screw/K-wire $(n=2)$, or a PDS suture $(n=1)$. Compression screws have been used significantly more often in the latter half of the study period $(\mathrm{p}=<0.01)$. Two complications were documented: in 1 patient operative revision and screw osteosynthesis became necessary due to mispositioning of the medial epicondyle during initial surgery using $\mathrm{K}$-wires. One patient had secondary dislocation during conservative treatment developing a pseudarthrosis without functional impairment. Sixty-eight patients were immobilized in an above-the-elbow cast for 29 days (range 11-50). The remaining eleven patients had early active mobilization using a joint motion device. Mean follow up was 7.6 months (2-61). Loss of elbow movement (LOM) was found to be mild in 30 and moderate in 15 patients. LOM was found to be associated with surgical treatment $(p=0.001)$, and with concomitant elbow dislocations $(p=0.29)$. Persistence of ulnar nerve palsy or recurrent joint instability have not been described. 
Conclusion: In order to prevent joint instability or pseudarthrosis, the operative treatment rate for pediatric medial epicondyle fractures nowadays is high. Screw osteosynthesis represents the increasingly preferred method of choice, because early active mobilization is possible. The long-term results after isolated medial epicondyle fractures are good, however, an associated elbow dislocation constitutes a relevant comorbidity.

\section{The special avulsion fracture case report of a 13-year- old female patient with dorso-cranial hip luxation and Pipkin IV injury}

\section{S. Hettenhausen ${ }^{I}$, P. Delhey ${ }^{I}$, U. Gronwald ${ }^{I}$, I. Fichtel ${ }^{2}$}

${ }^{1}$ Krankenhaus Bietigheim-Vaihingen, Klinik für Unfallchirurgie und Orthopädie, Bietigheim-Bissingen, Deutschland, ${ }^{2}$ Krankenhaus Bietigheim-Vaihingen, Klinik für Unfallchirurgie und Orthopädie, Bietigheim-Vaihingen, Deutschland

Question: Femoral head fractures are rare injuries in childhood and adolescence. The fractures are often associated with hip luxation [1]. According to Pipkin the classification depends on the height of the fracture in relation to the ligamentum capitis femoris and further concomitant injuries.

Methods: A 13-year-old girl fell from a ladder from about 20 feet on the left side of her body. The X-ray of the left hip showed a dorsocranial hip luxation with a fracture of the femoral head and the acetabulum. In addition, there were distal radius fractures on both sides. The patient was immediately transferred to the operating room. Through an anterolateral approach, an open reduction was performed, as the CT scan showed multiple small bone fragments in the acteabulum (Fig. 1). The fracture of the actebulum wall was well positioned after reposition and left without osteosynthesis. The fracture of the femoral head distal to the ligament was fixed with screws.

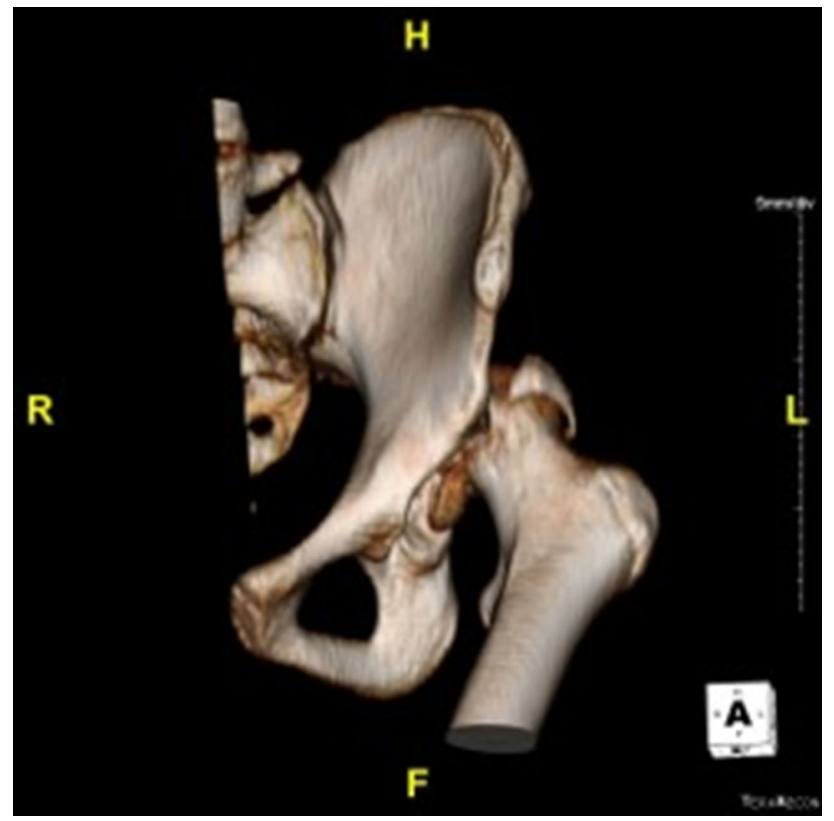

Fig. 1 3D reconstruction CT

Results: The patient walked with a limping gait on the left after 2 month, the internal rotation was only $15^{\circ}$. The MRI showed no evidence of femoral head necrosis or impingement. 6 months after trauma the mobility was $25^{\circ}-0^{\circ}-40^{\circ}$. The $\mathrm{X}$-rays showed a sclerosis of the acetabulum, as sign of consolidation of the fracture. The development of a posttraumatic arthrosis is actually not predictable. Conclusion: Hip dislocations with fractures of the femoral head and actebulum are rare events in childhood and adolescence. We were suprised, that the direct lateral fall on the greater trochanter led to the dislocation of the femoral head. Nehme et al. [2] also describe a anterior hip dislocation with dislocated actebulum fracture and greater trochanteric tear. They suppose that a direct lateral force on the greater trochanter can lead to anterior dislocation of the hip. In our case the lateral impact lead to a posterior dislocation. So a direct lateral impact on the greater trochanter seems to lead to femoral head dislocation either to anterior or posterior. According to Vaille et al. [1] a postoperative CT scan is not necessary. A wide joint space in $\mathrm{X}$-ray image should lead to surgical revision because of malalignment of the joint. A risk assessment between radiation exposure and surgical risk has not yet been investigated. Pipkin 4 fractures often lead to avascular necrosis [3]. In our case, the immediate open hip reduction and fixation resulted in good fracture healing.

References: [1] Vialle R, Odent T, Pannier S, Pauthier F, Laumonier F, Glorion C (2005) Traumatic hip dislocation in childhood. J Pediatr Orthop. 25(2):138-144.

[2] Nehme AH, Daoud JC, Abdelnour HG, Bou Mounsef JN, Moucharafieh RC, Wehbe JW (2017) Locked central fracture dislocation of the hip in a child after low-energy trauma. Case Rep Orthop 2017:6873484.

[3] Scolaro JA, Marecek G, Firoozabadi R, War JC, Routt MLC (2017) Management and radiographic outcomes of femoral head fractures. J Orthop Traumatol 18(3):235-241.

\section{Management of severely injured children in a paediatric trauma centre. Case report of two toddlers with combined injuries including high cervical spine injuries after motor vehicle accidents.}

\section{S. Reineke ${ }^{I}$, S. Kästner ${ }^{2}$, P. Illing ${ }^{1}$}

${ }^{1}$ Klinikum Kassel, Klinik für Kinderchirurgie und Zentrum für schwerbrandverletzte Kinder, Kassel, Deutschland, ${ }^{2}$ Klinikum Kassel, Klinik für Neurochirurgie, Kassel, Deutschland

Question: The treatment of severely injured pediatric patients is challenging for the responsible team. An interdisciplinary, motivated and cooperating team is vital for the safe and accurate diagnostics, therapy and psychosocial care of the young patients. It consists of physicians, nurses, social workers and psychologists experienced in treating injured children. The concept and structure of a pediatric trauma centre will be shown using the examples of two rare cases with injuries of the high cervical spine in children under 3 years of age after motor vehicle accidents.

Methods: Our interdisciplinary trauma team for paediatric patients, involving the centres for paediatric surgery, paediatric anaesthesia and intensive care, paediatric radiology, trauma surgery, neurosurgery, ENT and ophthalmology, treats patients from within a radius of 30-100 km and cooperating hospitals for basic and regular care. Results: Patient 1, 26-month-old, was hit by a car as a pedestrian. The boy was brought to our emergency room. The boy presented with a left distal femur fracture and an atlantoaxial dislocation. He was treated with overhead extension for the femur fracture and repositioning and immobilisation for the atlantoaxial dislocation. After 4 weeks the boy was discharged home without any neurologic signs. Patient 2, 35-month-old, was sitting behind the driver, restrained in a child safety seat during a high speed rear-end collision. The driver 
died on site. The girl was primarily admitted to another hospital and referred to us after 2 days for family reunion as her brother was treated in our hospital. During examination she reported pain in the neck region and right shoulder as well as an abnormal posture of the neck. The following radiological imaging showed a fracture of the right clavicle, a lesion of the ligaments of the dens axis as well as fractures of the thoracic vertebrae 7 and 9. The therapy was immobilisation of head, cervical and thoracic spine using a custom shaped Minerva brace. In addition, the family was supported by our psychologists to handle the demanding situation. After 2 weeks, the girl was discharged home without any neurologic signs. The hospital's internal data enquiry for the ICD-10 codes S13.11, S12.0 and S12.1 for the last 10 years showed only these two cases.

Conclusion: Both cases highlight the necessity of specialised paediatric trauma centres. Only they can provide the qualified expertise for treating severely injured children and adolescents in excellent and child-oriented manner. The shown injuries of the atlantoaxial region are dangerous, rare and characteristic for young children. Repositioning of the injury, if necessary, and external immobilisation are the columns of the predominantly conservative therapy.

\section{Fast-track therapy for chronic osteomyelitis in children-a concept for humanitarian aid in Germany?}

\section{A. B. Arif, J. Fester, N. Farhat}

Evangelisches Krankenhaus Hamm, Klinik für Kinderchirurgie und Kinderurologie, Zentrum für schwerbrandverletzte Kinder, Hamm, Deutschland

Question: The treatment of chronic fistulating osteomyelitis is associated with a long hospital stay and complex procedures. More and more hospitals in Germany are refusing to treat patients from humanitarian organizations free of charge for financial reasons and because of scarce resources. A concept to reduce hospital stay and consumption of resources in the treatment of chronic fistulating osteomyelitis in our hospital is presented. The aim of this concept is to achieve a higher level of approval by the hospital management in order to enable humanitarian aid maintaining patient safety.

Methods: In cooperation with Friedensdorf International, a nongovernmental organization (NGO) we selected 2 out of 11 patients. Inclusion criteria were: radiologically confirmed osteomyelitis, presence or absence of a fistula, no need for osteosynthesis. All diagnostic measures were carried out at Friedensdorf prior to admission. Inpatient admission was 1 day before surgery. Fistula excision, sequesterotomy, debridement, primary wound closure with a silicone flap for drainage and immobilization in a plaster splint for 2 weeks were performed. Patients received cefazolin $30 \mathrm{~min}$ prior to surgery, clindamycin i.v. until discharged and subsequent oral therapy for a total of 2 weeks. Dressing changes, gradual removal of the silicone flap within 1 week and X-ray control 4 weeks postoperatively were performed at Friedensdorf.

Results: In two cases we were able to reduce length of stay and minimize personnel, diagnostic and therapeutic resources by outsourcing preoperative work and postoperative care.

Conclusion: The presented fast-track therapy for chronic fistulating osteomyelitis in children is safe. Optimization of the treatment procedure leads to a significant cost reduction and may help to encourage and convince hospital management to allow treatment of children from developing countries.

\section{The use of Magnetix bioresorbable screws for limb fractures in childhood and adolescence. First experiances of a pediatric trauma center in Switzerland}

\section{T. F. Krebs, C. Matissek}

Ostschweizer Kinderspital, Kinder und Jugendchirurgie, St. Gallen, Schweiz

Question: Osteosynthesis with screws or wires is a common indication for fractures near the joint in children. After fracture consolidation usually the metal is removed in a second operation in general anesthesia. Over the last 10 years, intensive research has been carried out on bioresorbable implants. In Magnesium implants gas formation occurs due to the corrosion process and consists mainly of $\mathrm{H}_{2}$, CO and $\mathrm{CO}_{2}$. Gas formation leads to local osteolytic lesions which showed to have no clinical importance on fracture consolidation and stability. Still longterm effects on the growth plates are unknown. For this reason, epiphyseal crossing of the implants is contraindicated. We present our first experiences with magnesium screws (Syntellix, Germany) in 4 adolescents with fractures outside the growth plate, 1 metatarsal fracture \& 3 apophyseal avulsion fracture of the Epicondylus ulnaris.

Methods: Case 1: 12-year girl, supination trauma 13 days ago. Initially MT 5 fracture treated conservatively, assigned to us due to secondary dislocation. Mini-open reduction \& osteosynthesis by $3.5 \times 30 \mathrm{~mm}$ CBS screw. Case 2: $14 \mathrm{y}$ boy, fall on his right arm the day before X-ray picture shows $1 \mathrm{~cm}$ dislocated fracture of Epicondylus ulnaris. Open reduction \& osteosynthesis by $3.5 \times 40 \mathrm{~mm}$ CBS screw. Case 3: 12 y girl, fall on her right arm, X-ray picture shows $7 \mathrm{~mm}$ dislocated fracture of Epicondylus ulnaris \& Collum radii. Open reduction \& osteosynthesis by CBS $3.5 \times 40 \mathrm{~mm}$ (introperative break) \& CS $4.8 \times 60 \mathrm{~mm}$ screws. Collum radii only reposition. Case 4: 12 y girl, fall on her left arm, X-ray picture shows dislocated fracture of Epicondylus ulnaris. Open reduction \& osteosynthesis by CBS $3.5 \times 38 \mathrm{~mm}$ screw.

Results: Case 1: Uneventfull intra/postopperative course. Case 2: Uneventfull intraoperative course, break of screw at postop control (1 month postop.) with normal consolidation. Case 3: Intraoperative break of CBS screw, 2nd screw CS $4.8 \mathrm{~mm}$ with intra-/postoperative uncomplicated course/consolidation. Case 4: Uneventful intra/postoperative course.

Conclusion: We present our first experiences with magnesium based screws in pediatric fractures. Compared to conventional steel implants screw stability and tensile strength are to our eyes not equal yet but satisfying. Follow up controls showed 1 typical gas formation around the screw without negative effects for consolidation. 1 patient showed implant break 1 month postoperative with already consolidated fracture, 1 patient needed 2 screws due to intraoperative break of the first screw. In all 4 presented cases peri- and postoperative courses were otherwise uneventful, no further adverse side effects were observed.

Magnesium screws are a promising field, children/adolescents may benefit from biodegradable and stable implants even more than adults. In case of further positive studies bioresorbable implants could replace the "classic" implants. In addition to the individual medical benefit, the single-stage surgical procedure has also relevant economic benefits due to cost savings. 


\section{The atypical Monteggia-like lesion with both dislocated and fractured radial head in children}

\section{A. Herzog, T. Wirth, F. Fernandez}

\section{Olgahospital, Stuttgart, Deutschland}

Question: The atypical Monteggia-like lesion where the radial head is both dislocated as well as fractured represents a rare but challenging skeletal trauma in children. The severity of the injury and its impact are often underestimated, and therefore also often insufficiently treated. In this study we have evaluated our current management algorithm.

Methods: 17 cases of traumatic non-pathological atypical Monteggialike lesion with both dislocated as well as fractured radial head and ulnar shaft fracture that was treated primarily at our department during a period of 12 years between 2008 and 2019 were included in this study. Both the dislocated radial head fracture and the ulnar shaft fracture were managed by closed reduction and intramedullary stabilization. Furthermore, a postoperative immobilization was applied for 10 days by using a long arm cast. Radiological exams have taken place at cast removal, upon consolidation and shortly before hardware removal. Clinical follow-ups have been continued 3 months after hardware removal and then annually until bone maturity was reached. Results: The demographic of the study cohort at the time of the initial injury spread between 3.4 and $11.7(6.5 \pm 2.0)$ years. There were three cases involved toddlers under the age of 4 years. Due to the not yet ossified radial head cartilage at this age, the radial head fracture was not shown in the initial X-rays and was first diagnosed during the arthrography that was performed intraoperatively. No neurovascular injury was observed. The consolidation took $5.5 \pm 1.4$ weeks to be achieved. No delayed consolidation or pseudarthrosis occurred. In one case, the radial head re-dislocated before consolidation. This was detected by the radiological exam performed at the cast removal after 10 days and was revised by a second time of closed reduction and intramedullary stabilization. No other secondary displacement or correction loss, no material failure occurred before implant removal. The hardware removal was performed after $16.6 \pm 7.0$ weeks. No infection or relevant soft tissue irritation occurred before hardware removal. No premature hardware removal was necessary. Post-traumatic proximal radioulnar synostosis occurred in one other case, where a surgical resection became necessary and was combined with the hardware removal. Very satisfying clinical functional outcomes were observed at the follow-up 3 months after the hardware removal with an average Mayo Elbow Performance Score (MEPS) of $97.1 \pm 5.6$, where an excellent clinical functional outcome (MEPS > 90) was achieved in 16 cases and a good one (MEPS 75-89) in the other case.

Conclusion: Closed reduction with intramedullary stabilization has been shown as a valid method in the surgical management of the atypical Monteggia-like lesions with both dislocated and fractured radial head in children. X-rays alone are diagnostically not sufficient in toddlers due to the not yet ossified radial head cartilage. Therefore an intraoperative arthrography is mandatory for the correct diagnosis in these cases. A postoperative immobilization with radiological exam at the cast removal is necessary due to the risk of secondary radial head re-dislocation. However, only a short immobilization period of 10 days is recommended since prolonged immobilization are considered to be associated with increased risk of post-traumatic proximal radioulnar synostosis. Excellent clinical functional outcomes can generally be achieved by anatomical reduction and sufficient stabilization. Clinical follow-ups should be continued until bone maturity due to the dynamic nature of the humeroradial joint during the growth period.
Diagnostic tools and treatment decisions in peripheral nerve injuries of the upper extremity: how we do itthe St. Gallen approach

\author{
C. Matissek ${ }^{1}$, P. J. Broser ${ }^{2}$
}

${ }^{1}$ Ostschweizer Kinderspital, Kinder- und Jugendchirurgie, St. Gallen, Schweiz, ${ }^{2}$ Ostschweizer Kinderspital, Klinik für Neuropädiatrie, St. Gallen, Schweiz

Question: Peripheral nerve injury is frequently seen in supracondylar fractures and fractures of the forearm. Though transient and selflimited in many cases, there are severe lesions that require surgical intervention.

Methods: We present our diagnostic steps including neuropediatric and electrophysiological evaluation and the therapeutic implications in a selection of cases with fractures of the upper extremity.

Results: Neuropediatric assessment, including electrophysiology and nerve sonography, provides important information about peripheral nerve injury in terms of recovery potential and the need for surgical intervention.

Conclusion: In peripheral nerve injuries, an interdisciplinary approach helps to classify the severity of the nerve damage and to find optimal therapeutic strategies.

\section{Fractures of the proximal radius in children and adolescents: analysis of 100 consecutive cases}

\section{Dietzel, M. Schunn, S. Scherer, M. Esser, H. J. Kirschner, J. Fuchs, J. Lieber}

Department of Pediatric Surgery and Pediatric Urology, Children's University Hospital, Tübingen, Germany

Question: Fractures of the proximal radius only account for a small amount in pediatric forearm fractures. Therapy is conservative for non-displaced fractures and elastic stable intramedullary nailing (ESIN) can be an effective operative treatment option in displaced fractures of the radial neck. Our aim was to further investigate the treatment depending on the degree of displacement and to show possible pitfalls.

Methods: A retrospective data analysis of pediatric forearm fractures at our center between March, 2011 and December, 2019 was performed. We identified all fractures of the proximal radius and collected data concerning epidemiology, therapy, course and outcome.

Results: Between 03/2011 and 12/2019 one hundred patients [mean age 7.5 years $(1-15)]$ were found with a fracture of the proximal radius. Gender distribution was 62 girls: 38 boys; twenty-seven patients had concomitant injuries. Conservative treatment was performed in 63 patients (Judet $\mathrm{I}=27$; II $=30$; $\mathrm{III}=6$; Mason $\mathrm{I}=2$ ). They were immobilized in an above-the-elbow cast for 21 days (6-35). Operative treatment was performed in 37 patients (Judet $\mathrm{II}=3$; III $=22 ; \mathrm{IV}=5 ; \mathrm{V}=7$ ) using ESIN-osteosynthesis. An open surgical procedure for reduction was necessary in 5 cases. Additional plaster immobilization was performed in 32 cases. We documented 6 complications: loss of implant stability $(n=2)$, healing in malalignment, pseudarthrosis, radioulnar synostosis, and a persisting hypoesthesia at the thumb. Consequently, 2 ESIN osteosynthesis were revised and 1 radial head resection was performed due to loss of pro-/supination. At the latest follow-up loss of movement was seen in $11 \%$ of cases. The overall MEPI was 99.8 (90-100). None of the patients experienced negative impact on activities of daily life. 
Conclusion: Fractures of the proximal radius are predominately occurring without dislocation, and good results are obtained with conservative treatment throughout. However, in most cases the duration of immobilization is too long. In cases of radial neck fractures with unacceptable displacement, ESIN is the undisputed treatment of choice. However, we observed overtreatment and potential for technical failure with ESIN. Besides that, fractures profit from early mobilization and therefore plaster treatment is not indicated in addition to a stable osteosynthesis. Open surgery should be avoided whenever possible. Fractures of the radius head constitute as absolute rarities and therefore only case reports of adolescents are available in the literature.

\section{Chances and challenges of computational simulation of fracture healing in children}

\section{A. Lipphaus ${ }^{1}$, R.-B. Tröbs ${ }^{2}$,U. Witzel ${ }^{1}$}

${ }^{1}$ Ruhr-Universität Bochum, Forschungsgruppe Biomechanik, Bochum, Deutschland; ${ }^{2}$ Helios Klinikum, Klinik für Kinderchirurgie, Duisburg, Deutschland

Question: Computational simulation of fracture healing in adults have been reported to predict healing performance in accordance with clinical healing outcome. Mechanical stress thresholds are combined with biological rates of tissue differentiation to model the time course of fracture healing. This study investigates if existing healing algorithms can be adapted to simulate pediatric fracture healing by the use of age-dependent cell parameter rates.

Methods: A simplified model of a pediatric tibial shaft fracture (diameter $=19.5 \mathrm{~mm}$; marrow cavity $=12.5 \mathrm{~mm}$; gap size $=2.25 \mathrm{~mm}$ ) matching the dimensions of an 8 year old boy is created. Migration of mesenchymal stem cells and tissue differentiation are calculated based on previously established mechanobiological rules [1]. Halved population doubling time of stem cells in children compared to adults [2] is used while mechanical thresholds and differentiation time are assumed to be constant in both groups. Models with adult and pediatric process rates and interfragmentary movements of $0.6 \mathrm{~mm}$ known to lead to healing and of $2 \mathrm{~mm}$ causing non-unions are compared.

Results: Simulations with interfragmentary movement of $2 \mathrm{~mm}$ predicted non-union in adults and children. Time till first bony brigding of the fracture gap is 7 weeks for pediatric and 12 weeks for adult proliferation rates.
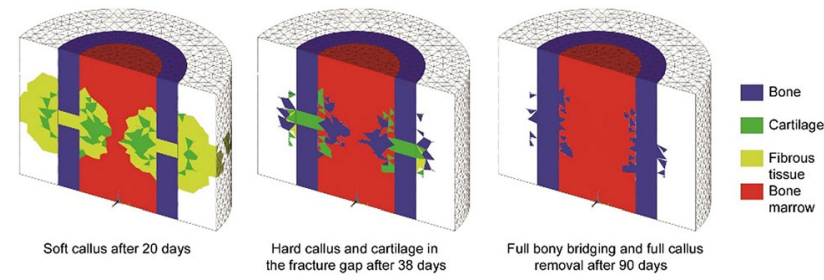

Soft callus after 20 days

removal after 90 days
reny bridging and fulc

Callus development and removal in children with $0.6 \mathrm{~mm}$ interfragmentary movement.

Conclusion: Mechanobiological algorithms of fracture healing in adults can be adapted to simulate pediatric healing. Higher proliferation is a possible reason for faster healing in children. Modeling of the complete bone including the epiphyseal plate and the periost, simulation of growth, and non-mechanical factors like metabolism, cytokines or angiogenesis is necessary. Validation of simulation results is possible by retrospect simulation of patient cases.
References: [1] Lipphaus A, Witzel U (2018) Finite-element syntheses of callus and bone remodeling: biomechanical study of fracture healing in long bones. The anatomical record 302: 2112-2121

[2] Marędziak M, Marycz K, Tomaszewski KA, Kornicka K, Henry BM (2016) The influence of aging on the regenerative potential of human adipose derived mesenchymal stem cells. Stem Cells International

\section{Is the full-body MRI an alternative to full-body CT in pediatric polytrauma diagnostics?}

S. Bakir ${ }^{1,2}$, M. Raimann
2,1 J. Ludwig $^{1}$, U. Rechenberg
,

${ }^{1}$ Unfallkrankenhaus Berlin, Klinik für Unfallchirurgie und Orthopädie, Berlin, Deutschland, ${ }^{2}$ Universitätsmedizin Greifswald, Klinik und Poliklinik für Unfall-, Wiederherstellungschirurgie und Rehabilitative Medizin, Greifswald, Deutschland, ${ }^{3}$ Unfallkrankenhaus Berlin, Klinik für Radiologie und Neuroradiologie, Berlin, Deutschland

Introduction: So far, there is no standard diagnostic procedure on the treatment of severely injured children, although severe accidents continue to be the leading cause of long-term impairment and death in childhood. In our level one trauma center, full-body MRI (fbMRI) has been implemented for high-energy trauma diagnostics in children as an alternative to full-body CT (fbCT). The aim of the retrospective study is to analyze to what extent the trauma diagnostics by fbMRI is a real alternative and which obstacles arise in comparison to the CT. Methods: The fbMRIs were performed between April 2007 and December 2018 in patients aged 0-16 years and compared with the fbCT scans performed in our clinic and at a second study site. Time latency up to the MRI/CT and the further radiological diagnostics were evaluated. In addition, the injury severity and any necessary analgesic sedation during the fbMRI were analyzed.

Results: 134 fbMRIs and 158 CTs were performed due to trauma at the two study sites. The groups showed comparable injury severity with a median Injury Severity Score of 5 [ISS range 0-24] for the MRI group and 6 [ISS range $0-75$ ] for the CT group. The median time between the patient's arrival and the MRI was 70 $\min$ [2-970 min], significantly longer $(p<0.001)$ than up to the CT with $26 \mathrm{~min}$ [3-367 min]. In $53.0 \%$ of the MRIs, additional radiological diagnostics were performed in advance (CT 30.4\%), while following the performed MRI, $67.9 \%$ received further diagnostics (CT 75.9\%). The number of patients who received analgesic sedation (possibly with additional intubation) was higher in the CT group with $37.3 \%$ to $21.6 \%$ in the MRI group. Of these patients, $86.4 \%$ (CT) and $27.6 \%$ (MRI) were already preclinically sedated $(\mathrm{p}<0.001)$. Correspondingly, $72.4 \%$ of the patients were first sedated in-hospital for MRIs. Conclusion: The fbMRI is an alternative and completely radiationfree diagnostic method for high-energy-traumatized children with hemodynamical stability. However, there are also limitations since due to the longer duration of the examination, additional analgesic sedation for the fbMRI was required much more frequently in contrast to the CT group. Probably due to the longer time to fbMRI, more radiological diagnosis was supplemented in advance. Overall, radiological diagnostics were often supplemented in both groups. Further studies should be performed to determine whether there is also an indication for fbMRI in circulatory unstable children. 
Motorized intramedullary lengthening nails can be an alternative to external fixators for distraction osteogenesis in children and adolescents
A. Frommer ${ }^{I}$, G. Toporowski ${ }^{1}$, G. Gosheger ${ }^{2}$, A. Laufer ${ }^{I}$, B. Bröking ${ }^{1}$, A. Rachbauer ${ }^{1}$, R. Rödl ${ }^{1}$, B. Vogt $^{1}$
${ }^{1}$ Pediatric Orthopaedics, Deformity Reconstruction and Foot and Ankle Surgery orthopaedics, deformity correction, foot and ankle surgery, University Hospital of Muenster, Germany; ${ }^{2}$ General Orthopaedics and Tumour Orthopaedics, University Hospital of Muenster, Germany

Question: This study examines the applicability of motorized intramedullary lengthening nails (ILN) for limb lengthening in children and adolescents. While this procedure represents an established method in the adult age group, limb lengthening with ILNs is still uncommon in children. In this juvenile age surgical lengthening procedures may be indicated for posttraumatic, congenital or idio- pathic leg length discrepancies. Compared to lengthening with external fixators complications such as pin site infection and soft tissue tethering can be avoided by using ILNs. However, when treating skeletally immature patients, surgeons encounter anatomical limitations due to shorter and thinner bones and need to account for remaining growth.

Methods: A retrospective clinical and radiographic analysis was conducted of all patients between the age of 8-16 years who were treated with ILNs at our orthopaedic department between 2016 and 2018.

Results: 60 segments of 54 patients (mean age at surgery 13.8 years) treated with ILNs were identified. The nails were implanted either by an antegrade femoral $(n=42)$, retrograde femoral $(n=10)$ or antegrade tibial $(\mathrm{n}=8)$ approach. In $58 / 60$ patients $(97 \%)$ the desired amount of lengthening was achieved with a mean distraction distance of $45 \mathrm{~mm}$. Due to osteitis in one patient and knee dislocation in another patient lengthening with ILN was prematurely ended. No complication related to the nailing approach was observed.
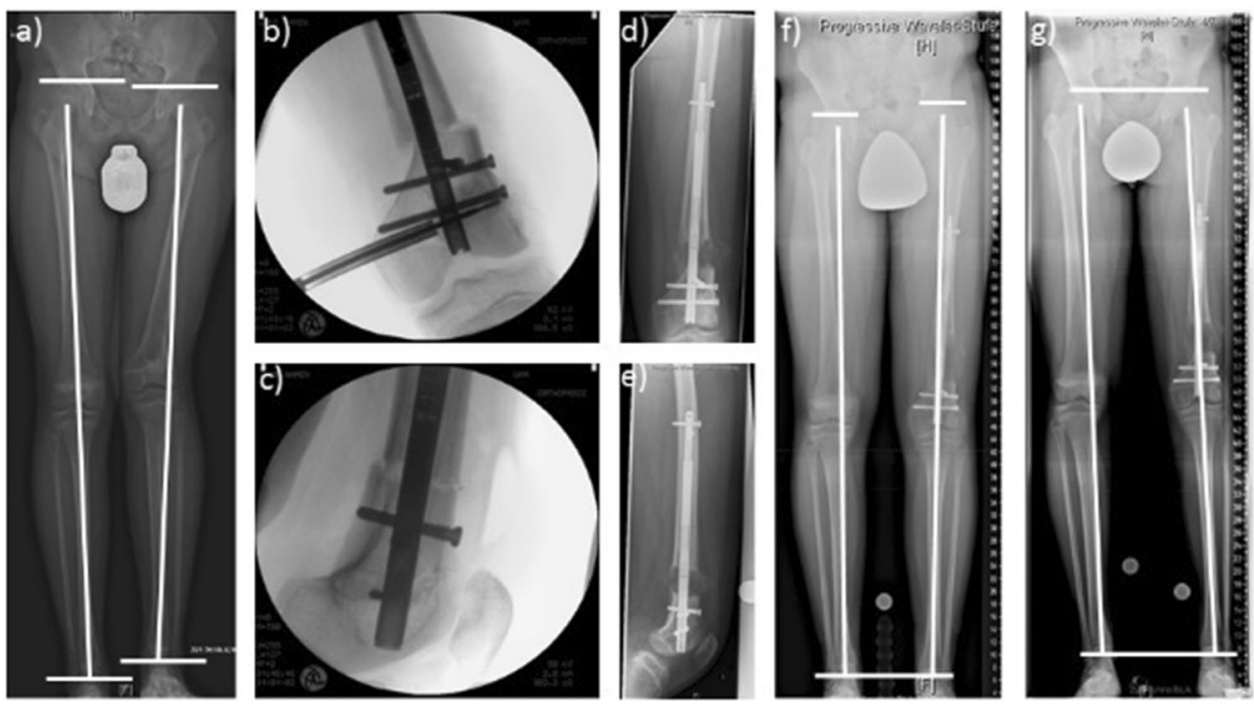

a) 13 year old boy with posttraumatic LLD of $3,8 \mathrm{~cm}$ and genu valgum. b, c) Retrograde femoral implantation of a PRECICE ${ }^{\circledR}$ lengthening nail and concomitant epiphysiodesis plus ad-hoc deformity correction. d, e) Lengthening with $1 \mathrm{~mm} /$ day. f) Planned overcorrection of the leg length discrepancy while the patient is still growing. g) Equal leg length and axis correction at the end of treatment. 
Conclusion: In selected skeletally immature patients, distraction osteogenesis with ILNs can reliably be applied. ILN insertion via an antegrade tibial approach only be considered for patients with preexisting damage to the growth plate or neglectable residual growth of the epiphysis. A minimum of $16 \mathrm{~cm}$ bone length and $17 \mathrm{~mm}$ diameter are required to insert the smallest available Precice ${ }^{\circledR}$ ILN (Nuvasive Specialized Orthopaedics, CA). To date, further studies are still required to investigate the long-term outcome.

\section{Treatment of fingertip amputations with a novel silicone fingercap: how surgeons can develop and test suitable medical devices themselves}

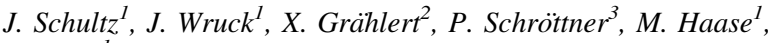 \\ G. Fitze l $^{l}$
}

${ }^{1}$ Universitätsklinikum Dresden, Klinik und Poliklinik für Kinderchirurgie, Dresden, Deutschland, ${ }^{2}$ Technische Universität Dresden, Medizinische Fakultät, Koordinationszentrum Klinische Studien, Dresden, Deutschland, ${ }^{3}$ Technische Universität Dresden, Medizinische Fakultät, Institut für Mikrobiologie und Hygiene, Dresden, Deutschland

Question: The outstanding potential of human fingertips to de novo form fully functional soft tissue and skin after amputations is known for decades. The standard treatment of these injuries has become selfadhesive film dressings.

Can treatment of fingertip amputation injuries be optimized while ensuring convenient and easy handling, wearing comfort and the best possible regeneration? Can pediatric surgeons develop medical devices without industrial partners that best fit pediatric needs and test this device in an investigator initiated RCT while meeting all ethical and legal standards in the highly regulated field of medical devices? Methods: We developed a finger cap, which can be attached to the finger without additional adhesives. It generates a protected space around the fingertip and an integrated wound fluid reservoir can be punctured for diagnostic and scientific purposes or the injection of pro-regenerative drugs in the future. It simultaneously prevents excessive leakage of putrid and malodorant wound fluid at the base of the finger. After patenting the silicone cap, all ethical and legal requirements following the German Medical Device Act were met to subject the finger cap to a randomized controlled pseudo-cross-over trial in which 22 patients were treated. 11 patients began treatment wearing the finger cap for 2 weeks, then they changed to self-adhesive film dressings for two additional weeks. The other half of the patients were treated in reverse order. After 4 weeks of treatment all patients could choose between to continue treatment with the silicone cap or the self-adhesive film dressing. Clinical data for each patient was collected weekly, including detailed descriptions of proceeding wound healing documented by photographs. Samples of wound fluid were gathered for future analysis of the proteome and the microbiome. 20 sets of patient data could be analyzed according to the study protocol.

Results: We included $4 \times$ Allen I, $10 \times$ Allen II, $6 \times$ Allen III, $2 \times$ Allen IV amputations. Patients were 2-72 years (average 38.2 years). Despite the prolonged treatment of $4-10$ weeks, the patients were pleased with the results, both in functional and cosmetic terms. A regular 2-point-discrimination and a nearly normal fingerprint were observed. Neither of the tested treatments led to severe complications. However, a loss of fingertip length an the formation of nail clawing were seen, when substantial amounts of the bony phalanx were amputated. Even injuries proximal of the flexor tendon insertion generated a satisfying outcome, however, without regeneration of the nail. 18 of 20 patients analyzed (90\%) chose the silicone finger cap as the preferred treatment.

Conclusion: We managed to develop and test a medical device for the treatment of fingertip injuries that best meets the needs of patients and clinicians. We achieved excellent clinical outcomes and gained wound fluid samples that will now be analysed for factors in the proteome and the microbiome that allow regenerative healing.

\section{Presciption behaviour for $\mathrm{X}$-ray use in pediatric and adolescent trauma patients}

\author{
Klaus Dresing ${ }^{1}$, Ralf Kraus ${ }^{2}$, Francisco Fernandez ${ }^{3}$, Peter \\ Schmittenbecher ${ }^{4}$, Kaya Dresing ${ }^{5}$, Christopher Spering ${ }^{1}$ und die \\ Sektion Kindertraumatologie der Deutschen Gesellschaft für \\ Unfallchirurgie
}

${ }^{1}$ Klinik für Unfallchirurgie, Orthopädie und Plastische Chirurgie der Universitätsmedizin Göttingen; ${ }^{2}$ Klinik für Unfallchirurgie und Orthopädie, Klinikum Bad Hersfeld; ${ }^{3}$ Kindertraumatologie, Klinikum Stuttgart Olgahospital. ${ }^{4}$ Kinderchirurgische Klinik, Städtisches Klinikum Karlsruhe. ${ }^{5}$ Darmstädter Kinderkliniken Prinzessin Margaret

Question: The indication for the (over)use of X-ray examination in pediatric and adolescent trauma patients should follow the ALARA principle. The radiation hazards and the effect of radiation on the growing sensible tissues of these young patient should always result in a strong indication for radiation use in diagnostics of injuries and controls after fracture care.

Methods: A detailed online survey of the section of pediatric traumatology (SKT) of the German Trauma Society (DGU) was open for 10 weeks. The target groups were trauma surgeons, pediatric surgeons, orthopedic surgeons and general surgeons working in or outside hospitals.

Results: Survey duration: 11/15/2019 to 02/29/2020. Participants 788. Participants' job profile: branch office $20.56 \%$, MVZ $4.31 \%$, hospital $75.13 \%$. Resident $16.62 \%$, senior physician $38.07 \%$ chief physician $22.59 \%$. Advanced training: $38.34 \%$ surgery, $33.16 \%$ trauma surgery, $36.66 \%$ special trauma surgery, $70.34 \%$ orthopedics and trauma surgery, $18.78 \%$ pediatric surgery. Frequency of contact with fractures in the above age group 36\% $<10$ /month, $27 \%<20$ / M, $36 \%>20 /$ M. $52 \%$ X-ray always in 2 planes after acute trauma. $\mathrm{X}$-ray of the opposite side $2.5 \%$ frequently. Ultrasound regularly used in diagnosis by $23 \%$. In polytraumatized children and adolescents $18 \%$ never use whole body CT, $50 \%$ rarely $14 \%$ use it as standard. Intraoperatively, collimation is always used by $50 \%$, postprocessing for magnification in $40 \%$, pulsed radiography is used by $47 \%$, and $89 \%$ do not use continuous fluoroscopy. 63\% never perform osteosynthesis directly on the image intensifier. Radiographic controls after metal removal are never used by $24 \%$. After operated supracondylar humerus fracture, different frequencies of controls up to 6 times are reported. After greenstick fracture of the distal radius, $40 \%$ refrain from further radiographic checks. In case of conservative treatment of a clavicle shaft fracture, 55\% refrain from any further $\mathrm{X}$-ray control. For an undisplaced tibial shaft fracture treated conservatively, $63 \%$ recommend radiographic control after 1 week in two planes, $24 \%$ after 2 weeks, $37 \%$ after 4 weeks, and $32 \%$ after 6 weeks.

Discussion: Analysis reveals that there is no consistent radiographic management of children and adolescents with fractures among respondents. In some cases, the indication for the use of X-rays does not appear to be justified. The ALARA principle does not seem to be universally observed. 
Conclusion: Comparing the documented results of the survey with the consensus results of the SKT we will need much persuasive efforts to change the radiation behaviour of the medical community.

\section{Hip spica cast immobilization in the treatment of femoral shaft fractures in small children}

\section{A. Herzog, T. Wirth, F. Fernandez.}

Olgahospital, Stuttgart, Deutschland

Question: With an incidence of 2\%, femoral shaft fractures represent one of the most common skeletal injuries in childhood. However, its optimal treatment strategy, especially in small children, has continued to be heavily debated. The purpose of this study is to evaluate the outcomes of femoral shaft fractures in small children that primarily treated through hip spica cast immobilization.

Methods: We have reviewed 72 cases involving traumatic, nonpathological, femoral shaft fractures in small children aged under 3 years $(1.9 \pm 0.8)$ old that were treated primarily through hip spica cast immobilization at our department between 2008 and 2018. A closed reduction was performed in the cases with a initial displacement of either over $10^{\circ}$ of varus or over $5^{\circ}$ of valgus in the frontal plane or either an antecurvation or a retrocurvation over $10^{\circ}$ in the sagittal plane or an axial shortening over $1 \mathrm{~cm}$ or a rotational malalignment. The hip spica cast immobilization was applied for 3 weeks. Radiological exams were performed after 10 days to check the fracture alignment in the cast and after 3 weeks upon cast removal to confirm the fracture consolidation. Routine follow-up exams were scheduled at 3 months and 15 months post-consolidation. In the cases with persistent malalignment, a further follow-up exam was performed at 6 years of age.

Results: All casts were applied within $24 \mathrm{~h}$ after admission. The average hospital stay lasted between 1 and 3 days $(1.5 \pm 0.6)$. In 4 cases, the hospital stay was prolonged due to minor adjustments of the cast prior to discharge. In 2 other cases, minor repair work was needed to maintain a proper immobilization. No reapplication of the cast under general anesthesia became necessary. No skin break down or neurovascular injuries were observed upon cast removal. One case of secondary displacement occurred before consolidation and was further treated through flexible intramedullary nailing. Nonunion was observed in none of the cases. Upon the follow-up exam at 3 months post-consolidation, full weight-bearing and free range of motion were achieved in all cases. In 5 cases, a persistent malalignment was still apparent upon the follow-up exam at 15 months post-consolidation, so that a further follow-up exam took place at the age of 6 , where no relevant leg length discrepancy over $15 \mathrm{~mm}(10.6 \pm 2.6)$ was observed.

Conclusion: The hip spica cast immobilization is a valid treatment option for femoral shaft fractures in small children. Temporary persistence of malalignment usually diminishes spontaneously within a reasonable time frame and the midterm leg length discrepancy generally remains under the tolerable upper limit. Comparably shorter hospital stay and higher subjective comfort are further advantages of the hip spica cast immobilization.

\section{Treatment standards to avoid disability after elbow injury}

\section{R. Kraus}

Klinikum Bad Hersfeld, Klinik für Unfallchirurgie und Orthopädie, Bad Hersfeld, Deutschland
Introduction: Fractures in the area of the elbow are frequent (approximately $15 \%$ of all fractures of the long bones) and often serious injuries to the growing musculoskeletal system. The growth potential of the elbow region is low, growth-associated axis corrections of posttraumatic malpositions can hardly be integrated into the treatment regimen. Even with optimal treatment, permanent consequences in form of axial deviations, instabilities, neurovascular disorders, but above all movement limitations are often left behind.

Methods: This presentation attempts to propose treatment standards for the most common injuries to the elbow region from the author's own experience and according to the current literature, which can provide extensive protection against unsatisfactory treatment results. Results: Supracondylar humerus fracture: Except for very young children, the therapeutic goal applies: to transfer each displaced fracture to an undisplaced fracture. At the time of the accident and after the treatment, subtle examination for neurovascular deficits and their documentation is necessary. When inserting osteosynthesis material, it must be ensured that nerve structures are not injured iatrogenic. After reduction and/or osteosynthesis careful analysis of the result in the X-ray image is undispensable: was an anatomical position (see therapy target) achieved? Short resting periods in the above elbow cast are to be applied depending on the stability of the operational supply. Physiotherapy to improve movement deficits only makes sense if they are not based on remaining bony misalignments. Lateral condyle fracture: patients are often very young $(<5$ years). In the X-ray image, the nucleus of the capitulum humeri is recognizable, but the trochlea is still only cartilageous! In the case of primary displaced fracture, open reduction is recommended. Open also means opening the joint to ensure that the joint line, which is not recognizable under image intensifier, is correctly reduced and free of steps. Osteosynthesis should be carried out with an ascending, if necessary crossing the growth plate, compressing screw. Without any or with insufficiently compressing osteosynthesis ( $\mathrm{K}$ wires), pseudarthrosis or radial overgrowth with subsequent cubitus varus may occur. In the case of primary undisplaced fracture, secondary dislocation must most likely be ruled out by an X-ray inspection after 4-5 days. Treatment of secondary displacement is done in the same way as for primary displaced fractures. Elbow dislocation with or without avulsion of medial epicondyle: elbow dislocation, with its torn ligaments, is a particularly serious injury to the elbow. If an avulsion of the medial epicondyle is present, it must be ensured that the fragment is not pinched into the joint. Screw osteosynthesis of the displaced epicondyle often provides sufficient stability of the joint in childhood and adolescence. Especially in adolescents, magnetic resonance imaging and, depending on the result, ligament reconstruction makes sense. Early functional exercise in the movement brace or in very unstable cases in the movement fixator is the basis of a good functional result. Monteggia lesion: with each supposedly isolated fracture of the ulna, the presence of an accompanying radius head luxation must be actively excluded. The radial head must be centered onto the capitulum humeri in each projection. If the radius head dislocation is diagnosed first, it is necessary to search for the underlying ulna injury. Immediately after the trauma, but latest 1 week after the trauma, the closed reduction of the radius head almost always succeeds by correcting the misalignment of the ulna. For osteosynthesis, the Elastic Stable Intramedullary Nailing (ESIN) is available on the shaft, with fractures located far proximal rarely even a plate or a drawstring belt. Long-time misdiagnosed radial head dislocations require far more complex procedures and do not certainly produce a good end result. Rare differential diagnosis is congenital radius head luxation, sometimes associated with radioulnar synostosis. Radial neck fracture: Fractures of the proximal radius are almost always slipped growth plates or metaphyseal fractures. Intraarticular fractures are almost non-present. Until about the age of 10, the correction potential for axis deviations of up to $45^{\circ}$ is very high. 
Prerequisite for the spontaneous erection of the radius head is an early functional exercise, which should begin after a week of resting for pain therapy. If the axial deviation goes beyond the abovementioned dimension, the manipulation should be carried out gently, as a closed reduction whenever possible. This, as well as stabilization, usually works very well with an intramedullary wire. Manipulating with a $\mathrm{K}$ wire in the fracture-site in sense of a Kapandji maneuver should be avoided, so as not to hurt the last still preserved periosteum. Only completely displaced fragments usually have to be reduced in an open manner.

Conclusion: This list does not claim to be exhaustive. However, if the proposed aspects are taken into account, in most cases a good longterm functional result can be achieved without serious long-term deficits.
Open Access This article is licensed under a Creative Commons Attribution 4.0 International License, which permits use, sharing, adaptation, distribution and reproduction in any medium or format, as long as you give appropriate credit to the original author(s) and the source, provide a link to the Creative Commons licence, and indicate if changes were made. The images or other third party material in this article are included in the article's Creative Commons licence, unless indicated otherwise in a credit line to the material. If material is not included in the article's Creative Commons licence and your intended use is not permitted by statutory regulation or exceeds the permitted use, you will need to obtain permission directly from the copyright holder. To view a copy of this licence, visit http://creativecommons.org/licenses/by/4.0/. 